Supporting Information

\title{
Experimental Evaluation of Kinetic and Thermodynamic Reaction Parameters of Colloidal Nanocrystals
}

\author{
Eric M. Brauser ${ }^{1,2 \#}$, Trevor D. Hul1 ${ }^{1 \#}$, John D. McLennan ${ }^{2}$, Jacqueline T. Siy ${ }^{3 *}$ and \\ Michael H. Bartl ${ }^{1 *}$ \\ ${ }^{1}$ Department of Chemistry, University of Utah, Salt Lake City, UT, 84112, USA \\ ${ }^{2}$ Department of Chemical Engineering, University of Utah, Salt Lake City, UT, 84112, \\ USA \\ ${ }^{3}$ Navillum Nanotechnologies, LLC, South Salt Lake, UT 84115, USA \\ ${ }^{\#}$ These authors contributed equally to this work. \\ *Corresponding authors: michael.bartl@utah.edu (MHB), j.siy@navillum.com (JTS)
}

\section{Varshni Coefficients}

Over the past two decades, the study of QD optical properties and their dependence upon crystal size have been extensively documented. Previous studies of bulk semiconductor materials and semiconductor nanoparticles have investigated the effect of temperature on the energy band gap. Through Varshni's relationship between the ground-state band gap and the effect of temperature on both crystal expansion and electron-phonon coupling, the characteristic energy which the QDs absorbs at room temperature can be extrapolated from observed measurements at elevated temperatures. In order to obtain accurate kinetic size data for each temperature, the measured absorption wavelength of the QD sample at room temperature was used with the first observed absorption wavelength of the kinetic study at elevated temperature. The value for the temperature coefficient $\alpha$ was then determined by fitting the Varshni equation with values of $\alpha$ and $\beta$. This reflects the temperature-dependent effects on the crystal structure that has been investigated and reported previously. ${ }^{\mathrm{S1}-\mathrm{S} 10}$ 
Real time measurement of the mean QD size using an in-situ absorbance cell allows for timeaccurate readings of the primary absorbance peak, and the corresponding size. With correction by the Varshni equation, a ground-state bandgap can be calculated, correcting for the observed wavelengths' dependence on temperature. ${ }^{\mathrm{S} 11}$

$$
E_{g}(T)=E_{0}-\frac{\alpha T^{2}}{T+\beta}
$$

The temperature coefficient $\alpha$ was calculated by fitting the Varshni equation for the bandgap shift at each elevated temperature. The measured room temperature (25 C) bandgap energy was 2.05 $\mathrm{eV}$ which was translated to a nanocrystal radius of $2.36 \mathrm{~nm}$ using the empirical relationship developed by Yu. et. al, shown in (4). ${ }^{\mathrm{S} 12}$

$D=\left(1.6122 * 10^{-9}\right) \lambda^{4}-\left(2.6575 * 10^{-6}\right) \lambda^{3}+\left(1.6242 * 10^{-3}\right) \lambda^{2}-(0.4277) \lambda+41.57$

The band gap energy for the initial point observed during each separate experiment was then input into the Varshni equation and, with input of the room temperature band gap energy and a slight rearrangement, provides a term with parameters $\alpha$ and $\beta$. Both parameters were optimized such that the $\Delta E$ term was minimized which provided a matrix of values. This matrix was then compared to literature values for validation. Figure $\mathrm{S} 1$ shows calculated results for $\alpha$, calculated for a single $\beta$. 


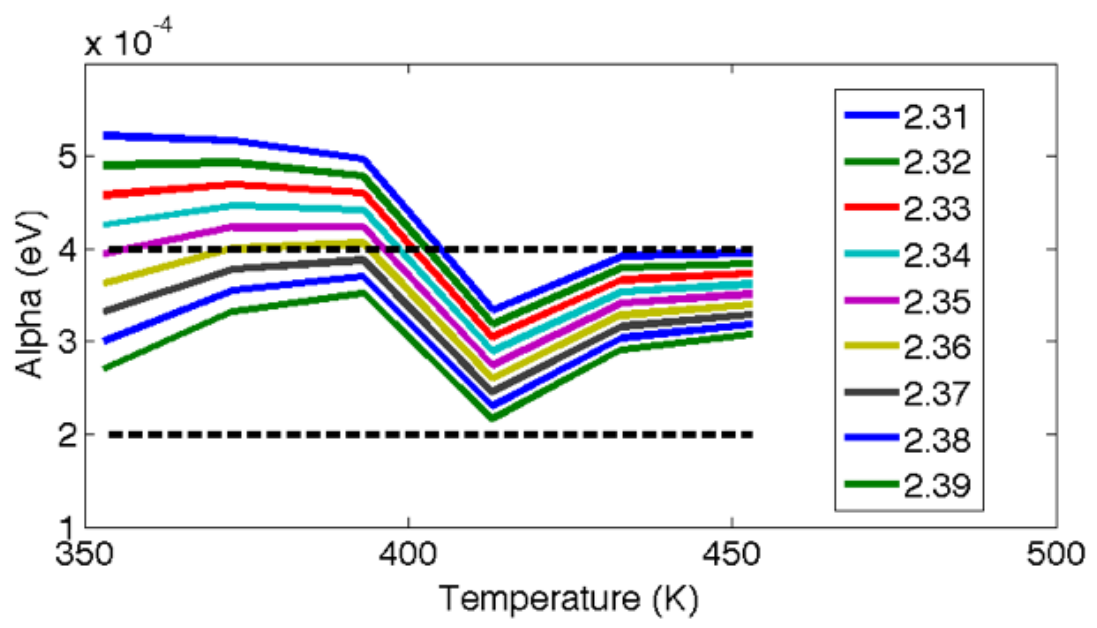

Figure S1: Alpha calculations for a range of potential room temperature radii.

Changing $\beta$ values within the range of reported literature values was observed to have very little impact on the minimization of $\Delta E$, so a value was chosen from Diroll et. al. that used similar components of study (CdSe/CdS) ${ }^{S 4}$ Note that the band gap shift in the Varshni equation is dependent on the input radius (corresponding to a bandgap energy and wavelength) and this radius has its own uncertainty. This necessitates that a range of initial radii $r_{o}$ need to be tested in the model in order to reflect possible "true" value of the radius. The dotted lines reflect high and low values of $\alpha$ for similar systems that have been reported in the literature. ${ }^{\mathrm{S} 1 \mathrm{~S} 6}$ As the temperature increases for each experiment, the calculated $\alpha$ decreases, which reveals the temperature dependence of $\alpha$. This is in agreement with reports that studied the effects of temperature variation on $\alpha$ for various semiconductor materials. ${ }^{\mathrm{S} 1, \mathrm{~S} 2, \mathrm{S4}, \mathrm{S6} 6}$ Therefore, a matrix of $\alpha$ values over the temperature range will be used to shift the observed radius for each experiment (as each experiment reflects dissolution at a different temperature). It is clear that as the input radius decreases, $\alpha$ increases beyond previously reported values across the temperature range. This reflects the inconsistency in trying to model parameters without constraining them within their physical meaning i.e. an unreasonable value for $\alpha$ would be required to reconcile an input radius of 2.31 or $2.38 \mathrm{~nm}$ with the observed initial radius. The dip in $\alpha$ at the $413 \mathrm{~K}$ mark is due to the initial observed radius at this temperature being smaller (larger bandgap energy) than the experiments at 393 or $423 \mathrm{~K}$. 
The overall trend however is a decrease in $\alpha$ across the temperature measurements. The $\alpha$ values for an input radius of $2.34 \mathrm{~nm}$ are near the reported literature values at low temperatures, and within the bounds at higher temperatures, in addition to showing minimal upward or downward trends. This radius is closest to that calculated from the QD sample absorption maxima wavelength at $602 \mathrm{~nm}$ calculated from (S2). For these reasons, the $\alpha$ values obtained from an input QD radius of $2.34 \mathrm{~nm}$ are shown in Table S1 and will be used for shifting the experimental data to reflect room temperature measurements.

Table S1: Modeled values of $\alpha$ across a range of QD dissolution temperatures

\begin{tabular}{|l|c|c|c|c|c|c|}
\hline Temp (K) & 80 & 100 & 120 & 140 & 160 & 180 \\
\hline$\alpha(\mathrm{eV} / \mathrm{K}$ E-4) & 4.260 & 4.463 & 4.418 & 2.891 & 3.537 & 3.618 \\
\hline
\end{tabular}

The Varshni correction method was also verified by quenching aliquots of QD samples from a given high-temperature treatment to room temperature. An absorbance spectrum was then taken at room temperature and the QD radius calculated from the wavelength position of the first excitonic absorption feature (as described above). The results were in agreement with Varshnicorrected high-temperature measurements of the same sample. 


\section{Damköhler Parameters}

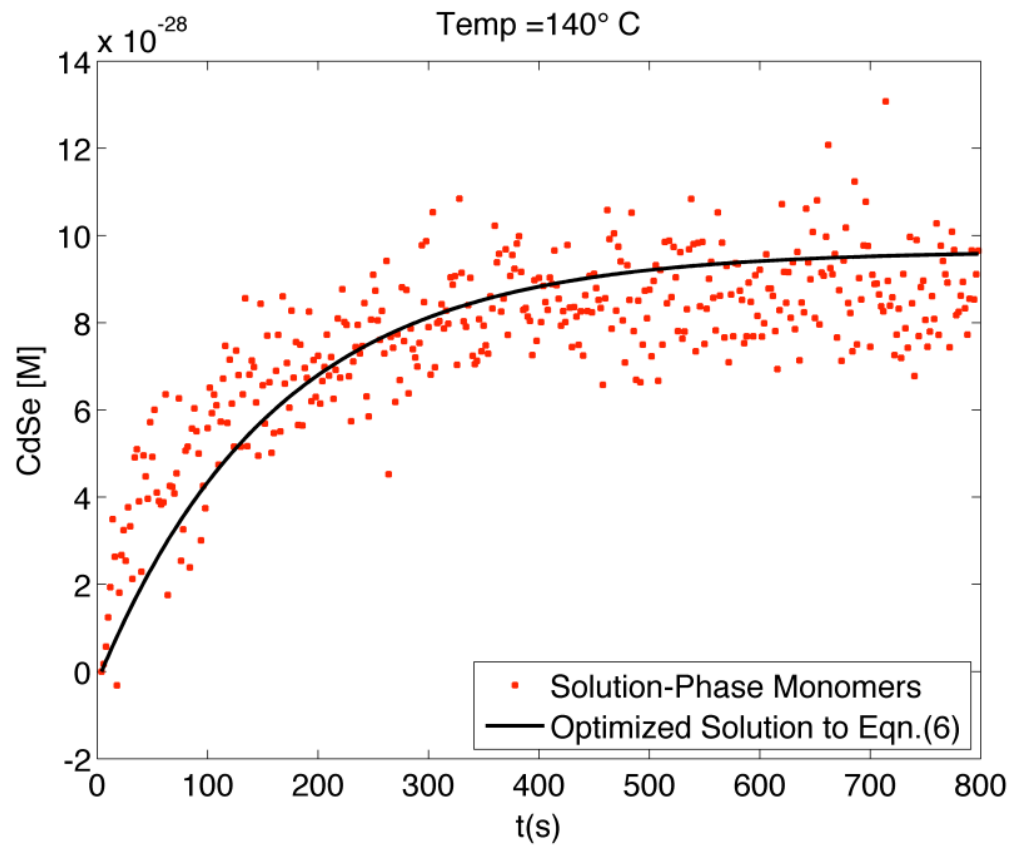

Figure S2: Example evolution of monomer concentration over time at $140{ }^{\circ} \mathrm{C}$ with optimized solution to equation (6), solving for mass transfer coefficient $k$ '. The monomer concentration was determined by the transfer of material from the solid to solution phase, calculated from the change in particle size.

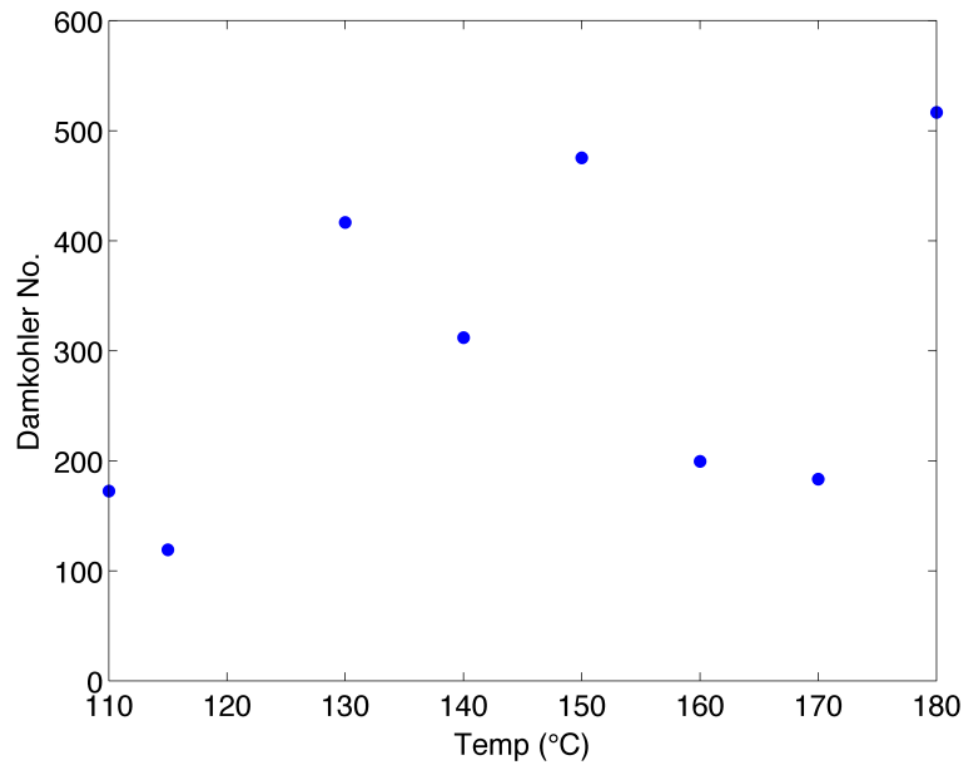

Figure S3: Plot of Damköhler parameter with temperature 


\section{Location of Characteristic Excitonic Wavelength Position}

The characteristic excitonic wavelength position was necessary to identify in order to obtain the mean QD radius from equation (S2). The QD solutions that were analyzed had low concentrations that resulted in non-ideal definition of the characteristic peak. Post-processing of the absorbance spectra was performed to improve the accuracy of locating the characteristic wavelength. A baseline following a fourth order polynomial was established to correct for scattering (Fig S4a). This baseline was only dependent upon the wavelength position, and therefore identical in shape between experiments. The baseline was subtracted from the experimental data, which was then fit to a Gaussian distribution (Figs. S4b and S4c). The center of this peak was used in equation S2 to determine the mean QD radius for subsequent time-series analysis. It is evident that this method provides a distinct peak and even with the poor resolution of the experimental absorbance spectra, the peak location can be confidently obtained. Note that the number of nanocrystals $N_{0}$ used in equation (3) is determined prior to dissolution, and the absorbance intensity over the course of the experiment is not a parameter necessary to successfully solve the differential equations.
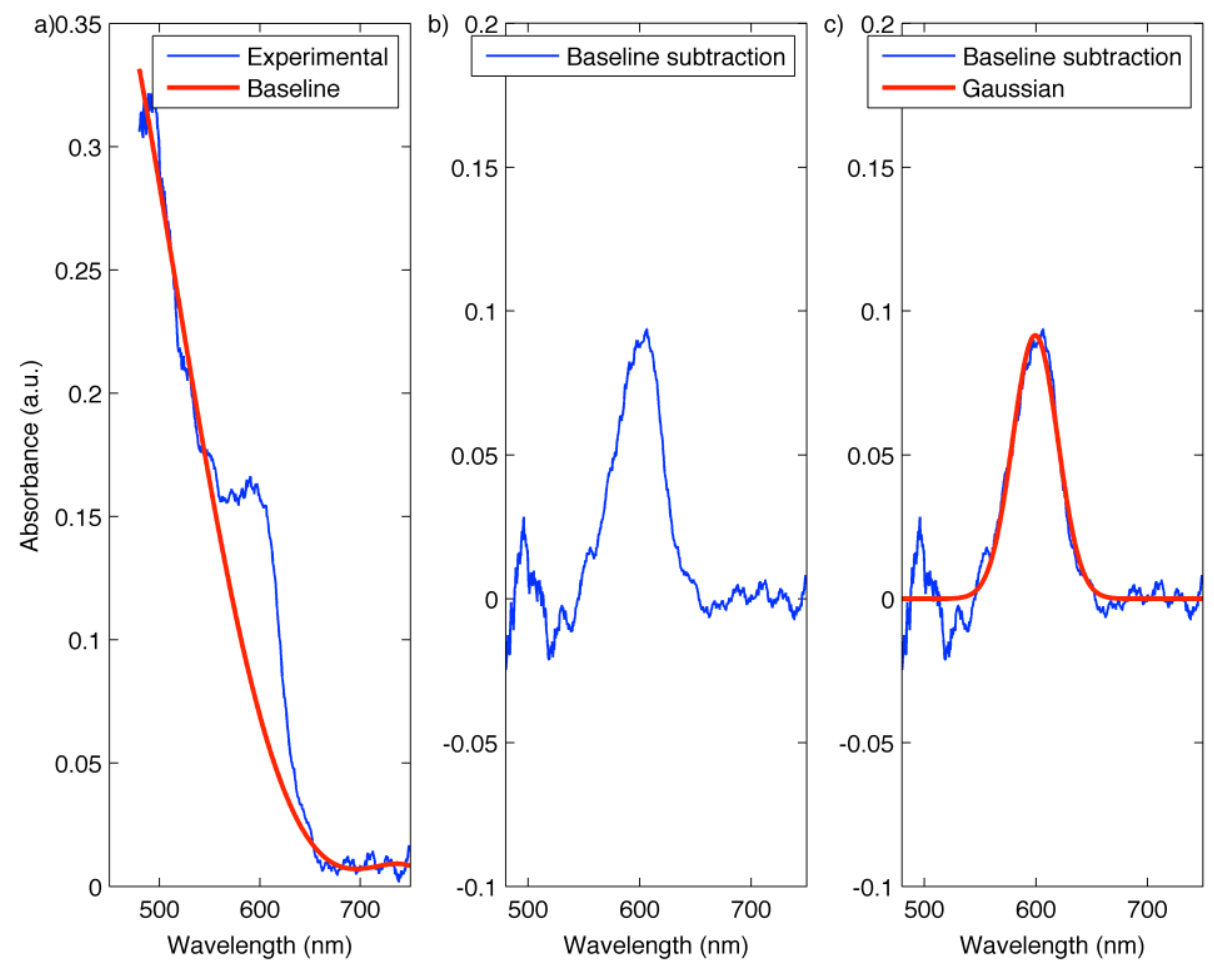

Figure S4: Gaussian distribution analysis of characteristic peak wavelength 


\section{Error Propagation}

We accounted for error through standard error propagation techniques, though the process was non-trivial. As stated in the manuscript, the Varshni equation was used to correct the hightemperature wavelength measurement to the room temperature value so that the temperature dependence would not influence the calculated radius values. This was done as follows:

1) Convert observed wavelengths to $\mathrm{eV}$

$$
\frac{\lambda_{2}}{1240 \mathrm{~nm}}=E_{2}[\mathrm{eV}]
$$

2) Use Varshni equation to convert the elevated temperature energy to equivalent room temperature energy

$$
E_{1}=E_{2}+\alpha\left[\frac{T_{2}^{2}}{T_{2}+\beta}-\frac{T_{1}^{2}}{T_{1}+\beta}\right]
$$

3) Use convert energy back to wavelength, and subsequently, using the Peng equation to obtain "true" radius

$$
\begin{aligned}
\lambda_{1}= & \frac{1240}{E_{1}}=1240 *\left[\frac{\lambda_{2}}{1240 \mathrm{~nm}}+\alpha\left[\frac{T_{2}^{2}}{T_{2}+\beta}-\frac{T_{1}^{2}}{T_{1}+\beta}\right]\right]^{-1} \\
D=(1.6122 * & \left.10^{-9}\right) \lambda^{4}-\left(2.6575 * 10^{-6}\right) \lambda^{3}+\left(1.6242 * 10^{-3}\right) \lambda^{2}-(0.4277) \lambda \\
& +41.57 \\
r=0.5 *\left(\left(1.6122 * 10^{-9}\right) 1240 *\left[\frac{\lambda_{2}}{1240 \mathrm{~nm}}+\alpha\left[\frac{T_{2}^{2}}{T_{2}+\beta}-\frac{T_{1}^{2}}{T_{1}+\beta}\right]\right]^{-1^{4}}\right. & \\
& -\left(2.6575 * 10^{-6}\right) 1240 *\left[\frac{\lambda_{2}}{1240 \mathrm{~nm}}+\alpha\left[\frac{T_{2}^{2}}{T_{2}+\beta}-\frac{T_{1}^{2}}{T_{1}+\beta}\right]\right]^{-1^{3}} \\
& +\left(1.6242 * 10^{-3}\right) 1240 *\left[\frac{\lambda_{2}}{1240 \mathrm{~nm}}+\alpha\left[\frac{T_{2}^{2}}{T_{2}+\beta}-\frac{T_{1}^{2}}{T_{1}+\beta}\right]\right]^{-1^{2}} \\
& \left.-(0.4277) 1240 *\left[\frac{\lambda_{2}}{1240 \mathrm{~nm}}+\alpha\left[\frac{T_{2}^{2}}{T_{2}+\beta}-\frac{T_{1}^{2}}{T_{1}+\beta}\right]\right]^{-1}+41.57\right)
\end{aligned}
$$


4) Now, error in radius can be determined via propagation:

$$
r=f\left(\lambda, T_{1}, T_{2}, \alpha, \beta\right) \text {, and }
$$

$$
\delta r=\left[\left(\frac{\partial r}{\partial T_{1}} \delta T_{1}\right)^{2}+\left(\frac{\partial r}{\partial T_{2}} \delta T_{2}\right)^{2}+\left(\frac{\partial r}{\partial \lambda} \delta \lambda\right)^{2}+\left(\frac{\partial r}{\partial \alpha} \delta \alpha\right)^{2}+\left(\frac{\partial r}{\partial \beta} \delta \beta\right)^{2}\right]^{0.5}
$$

This process rigorously calculates the error of the room temperature from measured values (temperature, wavelength) or uncertainty from earlier models (Varshni parameters). The uncertainty of the absorbance peak wavelength $\lambda$ is obtained from a Gaussian fit and accounts for the distribution of the QDs that contribute to the absorbance.

We found in this analysis that the error bars of the radii were quite large. For all except the highest temperatures, the error was greater than the radius shift from $r_{0}$ to $r_{\text {eq. }}$. Two examples, 160 and 180 ${ }^{\circ} \mathrm{C}$, are shown below:
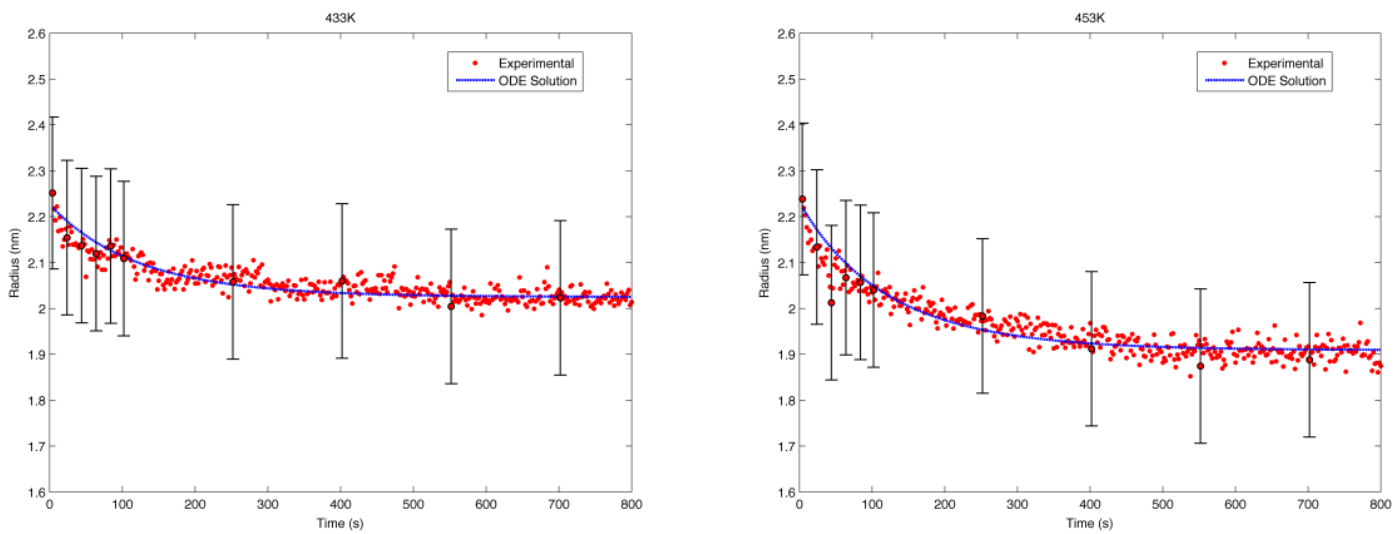

Figure S5: Data and ODE solutions from experiments conducted at 160 and $180{ }^{\circ} \mathrm{C}$, with black error bars determined by error propagation from individual uncertainty measurements

Clearly, even at the second-highest temperature, the initial radius is still within the uncertainty of the equilibrium radius. This method only takes into account the overall uncertainty of making a single measurement of the radius at elevated temperature, i.e. it is the overall uncertainty of the 
measurement technique. This is clear from the same values of uncertainty across the entire time range. To correct for this, the residuals from the model value were analyzed. The residuals for two experiments: 120 and $160{ }^{\circ} \mathrm{C}$, with dotted lines showing Gaussian 95\% confidence, are below:
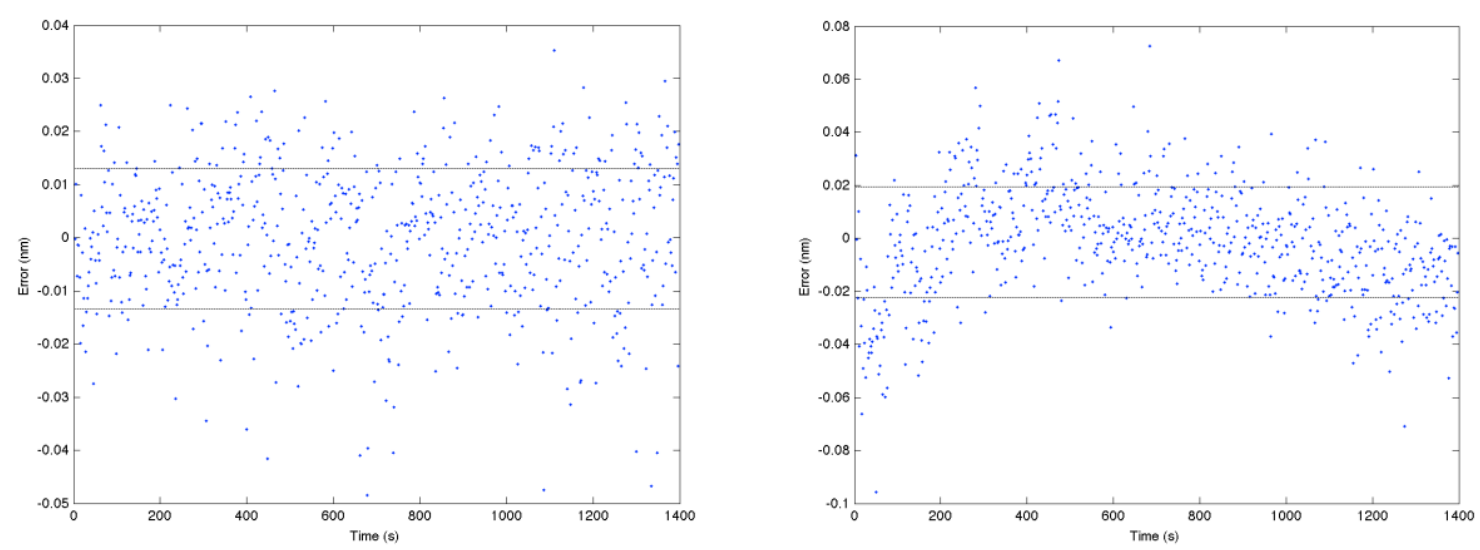

Figure S6: Time-resolved residuals of the solution set to the experimental data (blue dots). The black lines indicate the $95 \%$ confidence interval

At lower temperatures the residuals exhibit normal distribution. At high temperatures the errors are not normally distributed, because the short time solution changes rapidly. However the equilibrium distribution of residuals approaches a normal distribution, and is distorted by the inherent inability of a model to account fully for both short-time and long-time solutions. With these results, the variability of one observed radius value to a radius value at a previous time point is determined using the $95 \%$ confidence of the residuals. Subsequently, the error bars of the two temperature experiments are shown below: 

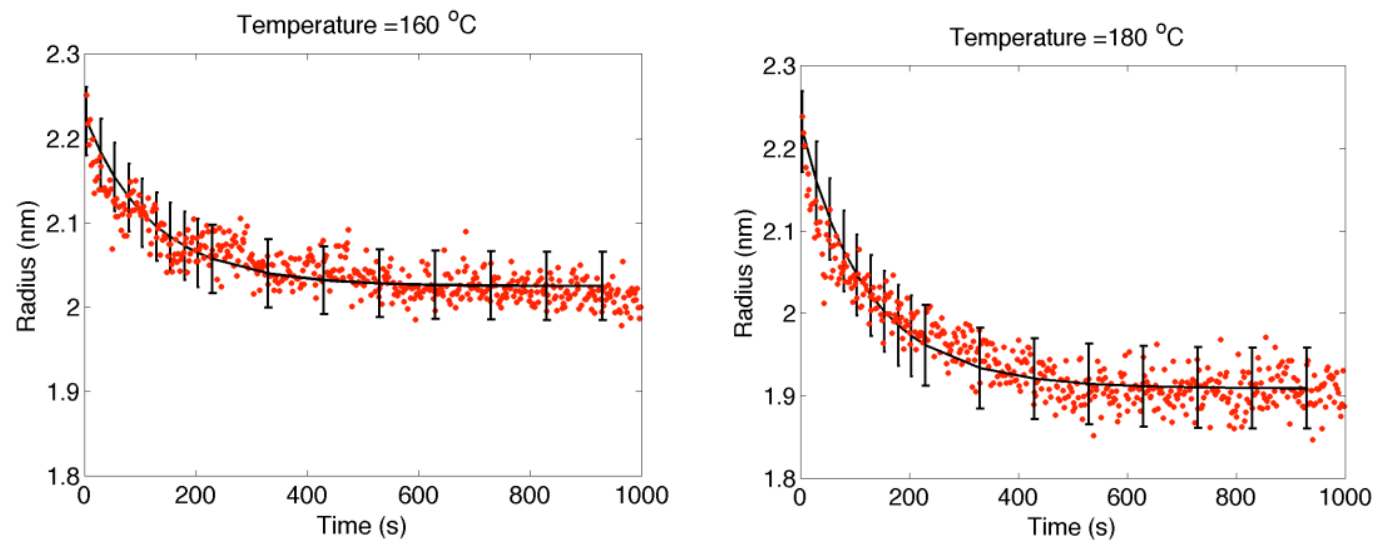

Figure S7: Data and ODE solutions from experiments conducted at 160 and $180{ }^{\circ} \mathrm{C}$ (red dots), with black error bars determined by the standard deviation of measurement variation from a previous point

Now, the initial radius is significantly different than those further in time at approximately 20-40 seconds for $180{ }^{\circ} \mathrm{C}$. Even at the lower temperature the initial radius is significantly different as soon as 100 seconds into the experiment. These results support our claim that the error in wavelength measurement and the Peng equation have not been ignored, but the error in the Peng equation is not appropriate for a time series measurement of dynamic nanocrystal size. Error bars for each set of experiments shown in figure 2 have been obtained.

\section{Linear Least Squares Fitting}

The experimental temperature-dependence data of the rate constants (growth and dissolution) and of the equilibrium constant were fitted to linearized Arrhenius and van't Hoff functions, respectively. It is important to note that uncertainties of measured data (e.g., readout of nanocrystal absorption wavelength position and absorbance, wavelength-to-radius conversion equation) are the same for the entire data set (all nanocrystal sizes and temperatures) and thus only shift the decay curves up or down (Figure 2 in manuscript). Such a shift, however, has no impact on the fitted rate constants for growth and dissolution. Therefore, uncertainties in the rate constant data result solely from the stability of the modeling solutions upon variation of input parameters and do not represent measured standard deviations. 
Two different fitting procedures were performed: simple linear least squares and weighted linear least squares fitting. While the obtained best estimates were within the uncertainty ranges, it should be emphasized that the theory of the weighted linear least squares method is based on the exact knowledge of the weights (defined as the inverse of the standard deviation squared, i.e. the inverse

of the variance). ${ }^{\mathrm{S} 13}$ For practical applications, this means that the weighted linear least squares method should only be used if weights can be estimated with high confidence. ${ }^{\text {S14 }}$ Since precise knowledge of experimental weights is missing for our data, the results from simple linear least squares fitting procedure were used for further analysis throughout this work.

\section{Complete Experimental Data for Thermodynamic Analysis}

The set of experimental data used for thermodynamic analysis, with overlain solutions to equation (3) from the manuscript, is shown below in figure S8. The kinetic parameters that were determined for these experiments were used in the subsequent thermodynamic analysis. Two things to note: 1) The results of the $120^{\circ} \mathrm{C}$ experiment were significant outliers and were not considered, and 2) The results from the two $180{ }^{\circ} \mathrm{C}$ experiments were averaged for the van't Hoff and Arrhenius plots (Figs. 3 and 4). Experiments conducted at temperatures lower than $110{ }^{\circ} \mathrm{C}$ do not exhibit significant size change between initial and equilibrium time, as seen in the $80{ }^{\circ} \mathrm{C}$ result in Fig. $2 \mathrm{~b}$. The $r(t)$ curves of these temperatures are almost flat, preventing the differential equation (3) to be solved properly. Therefore, these experiments were not considered for subsequent analysis and not shown below. 

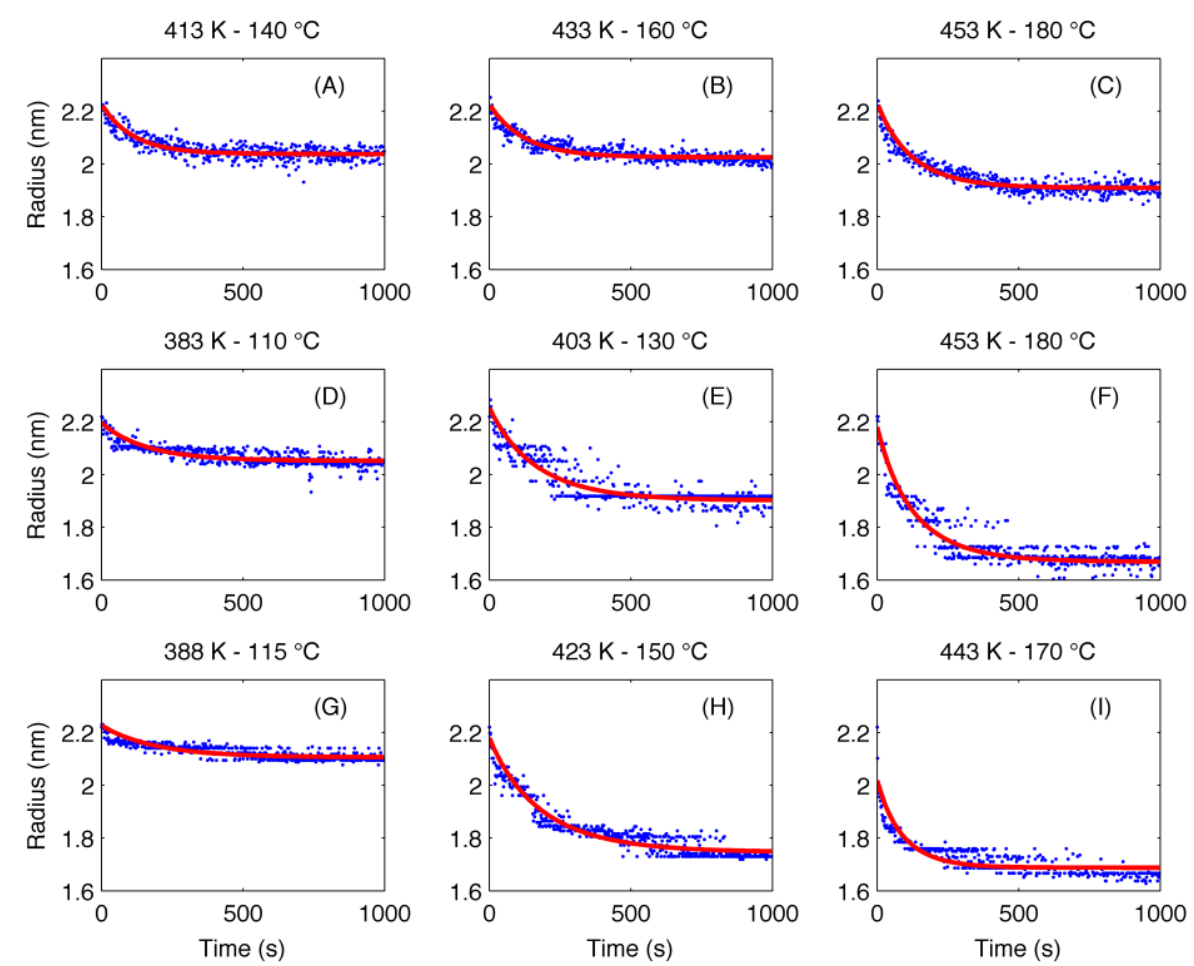

Figure S8: Data (blue circles) and ODE solutions (red lines) from experiments conducted from 110 to $180{ }^{\circ} \mathrm{C}$ that were used in the determination of the thermodynamic parameters. The figures are organized by original batch synthesis. Batch 1: figures $(A)-(C)$; Batch 2: figures $(D)-(F)$;

Batch 3: figures $(\mathrm{G})-(\mathrm{I})$.

\section{Estimated Concentration Profiles for Select Experiments}

Concentration values were calculated using the extinction coefficient method outline by $\mathrm{Yu}$ et

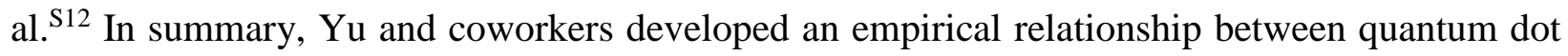
size and absorbance intensity for different materials ( $\mathrm{CdS}, \mathrm{CdSe}, \mathrm{CdTe})$. Figure $\mathrm{S} 9$ shows that the particle concentration over the course of the experiments is relatively stable. This an important result that was used to justify the assumption of constant concentration during the development of the governing equations, (3) and (4) in the manuscript. 


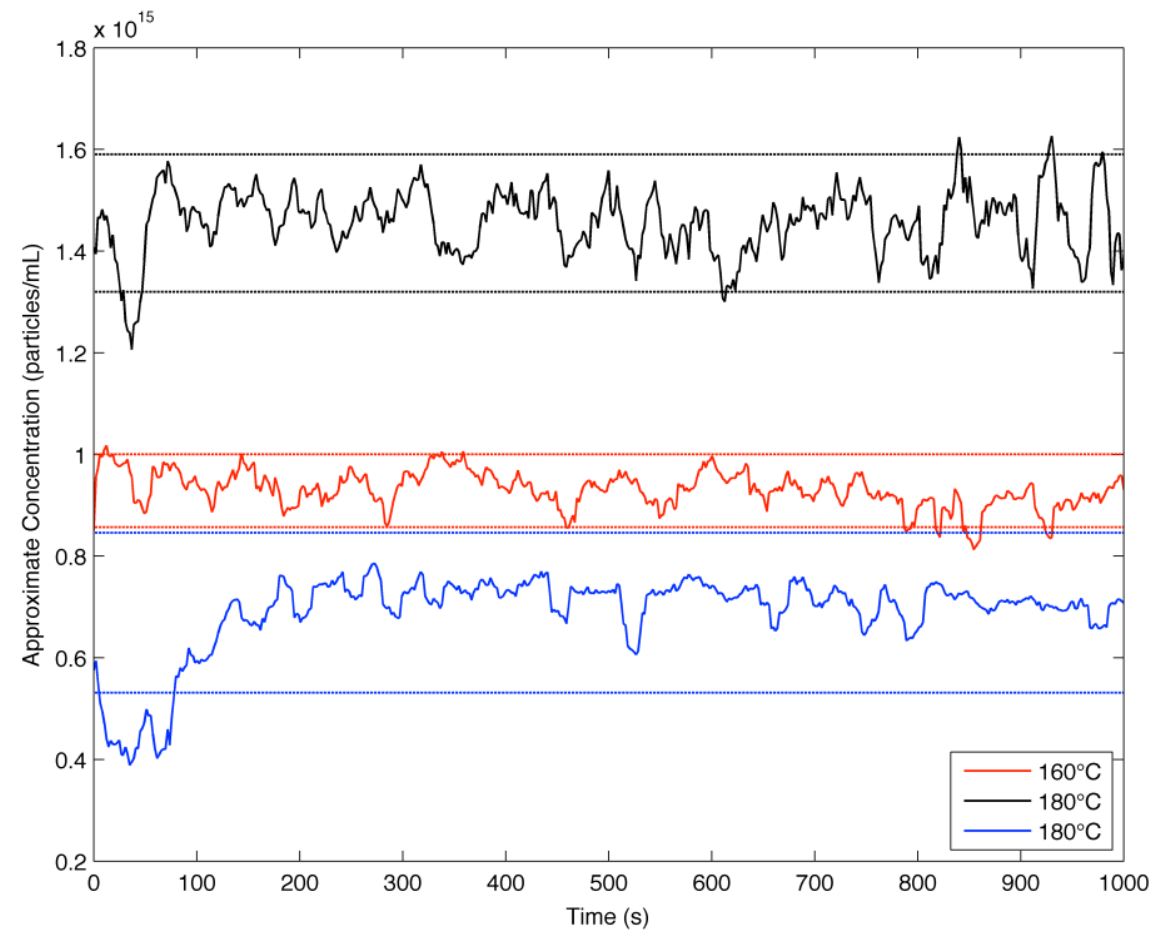

Figure S9: Concentration data calculated for 160 and two $180{ }^{\circ} \mathrm{C}$ experiments. Dotted lines indicate the $95 \%$ confidence interval.

\section{References - Supporting Information}

S1. Cheng, C.; Yan, H. Physica E 2009, 41, 828-832.

S2. Yang, P.; Chen, H.-S.; Zhang, S.; Zhao, J.; Du, Y.; Miao, Y.; He, H.; Liu, Y. RSC Adv. 2014, 4, 43800-43805.

S3. Rowland, C. E.; Schaller, R. D. J. Phys. Chem. C 2013, 117, 17337-17343.

S4. Diroll, B. T.; Murray, C. B. ACS Nano 2014, 8, 6466-6474.

S5. Dai, Q.; Song, Y.; Li, D.; Chen, H.; Kan, S.; Zou, B.; Wang, Y.; Deng, Y.; Hou, Y.; Yu, S.; Chen, L.; Liu, B.; Zou, G. Chem. Phys. Lett. 2007, 439, 65-68.

S6. Valerini, D.; Cretí, A.; Lomascolo, M.; Manna, L.; Cingolani, R.; Anni, M. Phys. Rev. B 2005, 71, 235409.

S7. Dubiel, M.; Brunsch, S.; Tröger, L. J. Synchrotron Radiat. 2001, 8, 539-541.

S8. Breivik, M.; Nilsen, T. A.; Fimland, B.-O. J. Cryst. Growth 2013, 381, 165-168. 
S9. Zheng, J.; Cao, S.; Wang, L.; Gao, F.; Wei, G.; Yang, W. RSC Adv. 2014, 4, 30948-30952.

S10. Iwanaga, H.; Kunishige, A.; Takeuchi, S. J. Mater. Sci. 2000, 35, 2451-2454.

S11. Varshni, Y. P. Physica 1967, 34, 149-154.

S12. Yu, W. W.; Qu, L.; Guo, W.; Peng, X. Chem. Mater. 2003, 15, 2854-2860.

S13. Ryan, T. P. Modern Regression Methods (Wiley, New York, 1997, pp. 60-70).

S14. NIST/SEMATECH e-Handbook of Statistical Methods, http://www.itl.nist.gov/div898/handbook/pmd/section1/pmd143.htm 